\title{
Aging does not aggravate the pregnancy-induced adaptations in glucose tolerance in rats
}

\author{
Silvia Caluwaerts*, Kathleen Holemans, Rita van Bree, Johan Verhaeghe, \\ F. André Van Assche \\ Department of Obstetrics and Gynecology, Katholieke Universiteit Leuven, 3000 Leuven, Belgium \\ Received 3 December 2004; accepted 13 October 2005
}

\begin{abstract}
Older age is an assumed risk factor for the development of gestational diabetes mellitus (GDM) in women. Here, we studied the effect of age and pregnancy on fat mass and glucose tolerance in rats. We performed intravenous glucose tolerance tests (IVGTTs) in 3- and 9-monthold rats, either nonpregnant or pregnant (day 20). In addition, we measured maternal fat mass, by dual-energy x-ray absorptiometry, and plasma leptin and lipid levels, as well as fetal parameters, on day 22. Nine-month-old rats had higher fat mass and plasma leptin, cholesterol, and triglyceride concentrations than 3-month-old rats. Glucose levels during the IVGTTs were elevated at several time points in 9-month-old rats, and the area under the curve (AUC) was increased. Pregnancy did not affect fat mass or the AUC for glucose during the IVGTT. The AUC for insulin during the IVGTTs was increased by age as well as pregnancy, but there was no interaction between the two by 2-factor analysis of variance. Reproductive performance was less optimal in 9-month-old rats, with a reduction of individual fetal and placental weight. In conclusion, 9-month-old rats exhibit a deterioration in glucose tolerance, possibly linked to the age-dependent increase in fat mass and leptin concentrations. Pregnancy also comprises certain adaptations in lipid and glucose metabolism, but because no interaction was found between both factors, the effect of pregnancy is not aggravated by aging. This may suggest than an increased gestational diabetes mellitus prevalence in older women can similarly be explained by age as such.
\end{abstract}

(C) 2006 Elsevier Inc. All rights reserved.

\section{Introduction}

The age at which women become pregnant has gradually increased over the last decades in Western countries, concomitant with the widespread use of assisted reproductive techniques. In 2001, about $14 \%$ of births in the United States were from women aged 35 years or older, which represents a 54\% increase compared with 1990 [1]. Maternal age older than 35 years is associated with an augmented risk of several pregnancy complications [2], including gestational diabetes mellitus (GDM) [3]. For example, GDM occurred in $20 \%$ of gravidas aged older than 50 years achieving a pregnancy by oocyte donation; within this group, GDM was more common among women aged 55 years or older $(40 \%)$ than among women aged 50 to 54 years $(13 \%)[4]$.

* Corresponding author. Tel.: +32 16 346195; fax: +32 16344205 .

E-mail address: silvia.caluwaerts@uz.kuleuven.ac.be (S. Caluwaerts).
Aging reduces glucose tolerance by affecting both insulin secretion and action. However, the intertwinement between age and adiposity makes interpretation less obvious; the deterioration in insulin action appears to be explained by increased body fat rather than by age itself [5]. Insulin secretion declines gradually with age [6] and is inappropriate for the degree of insulin resistance. In rats, aging is associated with a decreased insulin-dependent glucose uptake by soleus and heart muscle, diaphragm, and adipose tissue [7], and also with an impaired insulin secretory response, possibly mediated by a reduction in glucose transporter 2 expression in beta cells [8]. Ultrastructurally, beta cells of aging rats have abnormal mitochondria and lysosomes, and a reduced proliferative capacity [9]. These effects of aging may be intensified by the simultaneously increased secretion of adiposity-related cytokines in aging individuals. Leptin reduces glucose transport by abolishing glucose transporter 2 phosphorylation and inhibits insulin secretion in pancreatic beta-cell lines [10]. Similar in vitro experiments indicated that tumor necrosis factor $\alpha$ sup- 
presses both basal and glucose-stimulated insulin transcription and secretion [11].

Pregnancy reversibly modulates glucose metabolism. Insulin resistance is a hallmark of the second half of normal pregnancy, resulting in increased basal and stimulated insulin concentrations [12]. However, women who develop GDM show a $67 \%$ impairment of beta-cell compensation for insulin resistance [13]. Similarly, normal rat pregnancy is accompanied by a gradual insulin resistance at the liver and at peripheral tissues [14]. Beta-cell compensation occurs in young-adult gravid rats, characterized by increased betacell proliferation and insulin secretory capacity [15-17].

Here, we compared the effects of age on glucose tolerance in both pregnant and nonpregnant rats. We hypothesized that glucose tolerance during pregnancy would deteriorate in 9-month-old rats, as compared with 3-month-old rats. In addition, we measured fat mass by dual-energy x-ray absorptiometry (DXA).

\section{Materials and methods}

All experiments were reviewed and approved by the local ethics committee for animal procedures (K.U. Leuven, Belgium). Wistar rats, purchased from Charles River Laboratories (Wilmington, MA), were kept in controlled conditions of light (12 hours dark, 12 hours light) and humidity, were fed a standard laboratory chow ad libitum (Trouw, Gent, Belgium), and had free access to tap water. At the start of the study, rats were either 3 or 9 months old. All animals were weighed, and a nonfasting blood sample was taken from the tip of the tail to determine glucose, insulin, and leptin levels. Three- and nine-month-old rats were mated overnight just before estrus; the presence of spermatozoa in a vaginal smear taken the next morning confirmed their pregnancy (day 1).

\subsection{Intravenous glucose tolerance test}

Glucose tolerance was examined in 3- and 9-month-old dams, either nonpregnant ( $\mathrm{n}=8$ and 9 , respectively) or on day 20 of pregnancy ( $\mathrm{n}=7$ and 9 , respectively). On the day before the glucose tolerance test, rats were anesthetized with ketamine $50 \mathrm{mg} / \mathrm{kg}$ IP (Parke-Davis, Zaventem, Belgium), xylazine $10 \mathrm{mg} / \mathrm{kg}$ IP (Bayer, Leverkusen, Germany), and atropine $0.25 \mathrm{mg} / \mathrm{kg}$ IP (Sterop, Brussels, Belgium). The jugular vein was cannulated, and the catheter (Degania Silicone, Degania Bet, Israel) was tunneled to the back. The animals recovered overnight, and water was available ad libitum. The following morning, blood was collected by tail snipping to determine basal levels of glucose and insulin. A bolus of $1 \mathrm{~g} / \mathrm{kg}$ glucose $30 \%$ was injected intravenously. Blood glucose levels were determined on tail-prick blood samples after 5, 10, 15, 30, 60, and 90 minutes using a glucometer (Glucocard Memory 2 GT-1640, Menarini Diagnostics, Florence, Italy). At the 10-, 30- and 90-minute time points, a larger blood sample was also collected from the tip of the tail for the simultaneous measurement of plasma insulin. At the end of the procedure, rats were euthanized by a pentobarbitone overdose, and carcasses of nonpregnant rats were kept at $-20^{\circ} \mathrm{C}$ for analysis of body composition using DXA on a Hologic QDR-1000/W absorptiometer (Hologic, Inc, Zaventem, Belgium).

\subsection{Maternal data and fetal data on day 22 of intrauterine life}

On days 6, 13, and 20 of pregnancy, rats (3 months old, $\mathrm{n}=8 ; 9$ months old, $\mathrm{n}=9$ ) were weighed, and nonfasting blood samples were collected from the tip of the tail into heparinized tubes; the plasma was stored at $-20^{\circ} \mathrm{C}$ for the measurement of glucose and insulin concentrations. On day 22 of pregnancy, maternal rats were anesthetized with $0.24 \mathrm{mmol} / \mathrm{kg}$ pentobarbital IP (Sanofi, Brussels, Belgium), and fetuses were delivered by cesarean delivery. Fetal blood was collected through axillary incisions, with the fetuses still attached to the umbilical cord, and was pooled for all fetuses in each uterine horn (ie, 2 collections per litter). All fetuses and placentas were weighed, and fetal plasma was stored at $-20^{\circ} \mathrm{C}$ for measurement of glucose and insulin concentrations. Maternal blood was drawn from the aorta, and rat carcasses were kept at $-20^{\circ} \mathrm{C}$ for analysis of body composition using DXA. Glucose was determined using the glucose oxidase method with a YSI 2300 Stat Plus Glucose Analyzer (Yellow Springs Instruments, Yellow Springs, $\mathrm{OH})$. Insulin was measured by radioimmunoassay with rat insulin as the standard (Linco Research, St Charles, MO) and a guinea pig antirat insulin antibody, kindly donated by A Kervran (Paris, France). Leptin was measured by radioimmunoassay (Linco Research), and cholesterol and triglycerides by kits from Roche (Mannheim, Germany).

Table 1

Parameters measured in 3- and 9-month-old rats before and at the end of pregnancy

\begin{tabular}{|c|c|c|c|c|c|c|c|}
\hline & \multicolumn{2}{|c|}{3 mo old } & \multicolumn{2}{|c|}{9 mo old } & \multicolumn{3}{|c|}{ Two-factor ANOVA $(P)$} \\
\hline & $\operatorname{NPr}(\mathrm{n}=8)$ & $\operatorname{Pr}(\mathrm{n}=7)$ & $\operatorname{NPr}(n=9)$ & $\operatorname{Pr}(\mathrm{n}=9)$ & Age & Pregnancy & Interaction \\
\hline Body weight (g) & $265 \pm 3$ & $346 \pm 7$ & $325 \pm 5$ & $404 \pm 8$ & $<.0001$ & $<.0001$ & NS \\
\hline Glucose (mg/dL) & $124 \pm 4^{\mathrm{a}}$ & $81 \pm 3^{b}$ & $108 \pm 3^{\mathrm{a}}$ & $82 \pm 3^{b}$ & $<.05$ & $<.0001$ & $<.05$ \\
\hline Insulin (mU/L) & $28 \pm 2$ & $43 \pm 4$ & $41 \pm 5$ & $33 \pm 7$ & NS & NS & $<.05$ \\
\hline Leptin (ng/mL) & $3.4 \pm 0.3$ & $1.9 \pm 0.4$ & $6.5 \pm 0.6$ & $4.6 \pm 1.1$ & $<.0005$ & $<.05$ & NS \\
\hline Cholesterol (mg/dL) & $64 \pm 4.2$ & $47 \pm 3.0$ & $70 \pm 4$ & $64 \pm 4.5$ & $<.05$ & $<.05$ & NS \\
\hline Triglyceride (mg/dL) & $65 \pm 7.5$ & $90 \pm 18$ & $151 \pm 19$ & $236 \pm 40$ & $<.0005$ & .055 & NS \\
\hline
\end{tabular}

Number of animals is indicated in parentheses. All values are means \pm SEM. Bonferroni post hoc test indicated intergroup differences for glucose (denoted by different superscripts), whereas no differences were found for insulin. Pr indicates pregnant; NPr, nonpregnant. 


\subsection{Data analysis}

Data analysis was performed using GraphPad Prism software version 4.00 for Windows (GraphPad Software, San Diego, CA). The respective effects of age and pregnancy were studied by 2-factor analysis of variance (ANOVA). Because statistical power to detect interactions is lower than for main effects, we considered all interactions of "age" and "pregnancy" at a $P<.15$ level. If an interaction between the 2 factors was detected at the $P<.15$ level, we also performed 1-way ANOVA to detect overall differences between the 4 groups, followed-if significant $(P<.05)$ - by Bonferroni multiple comparison test to analyze intergroup differences. Analysis of glucose and insulin levels during intravenous glucose tolerance test
(IVGTT) was performed by calculating the area under the curve (AUC) for each animal. Correlations were examined by nonparametric Spearman correlation coefficients. Differences between 3- and 9-month-old rats were analyzed by a 2 -sided unpaired $t$ test and were considered significantly different when $P<.05$. All data were expressed as means \pm SEM.

\section{Results}

As expected, body weight was higher in 9-month-old rats than in 3-month-old rats (Table 1). In addition, 9-month-old rats had higher circulating leptin, cholesterol, and triglyceride concentrations than 3-month-old rats.

\section{NON-PREGNANT RATS}

A

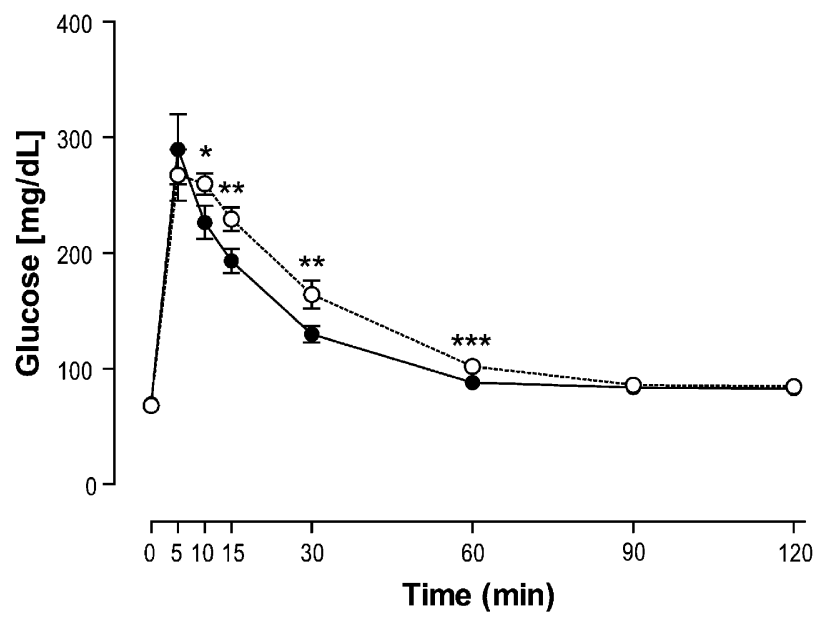

B

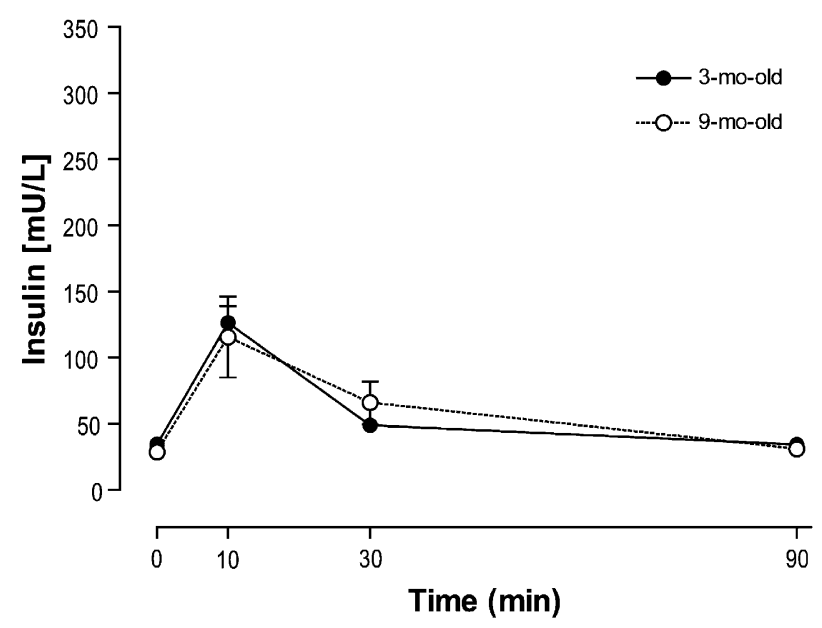

\section{PREGNANT RATS}

C

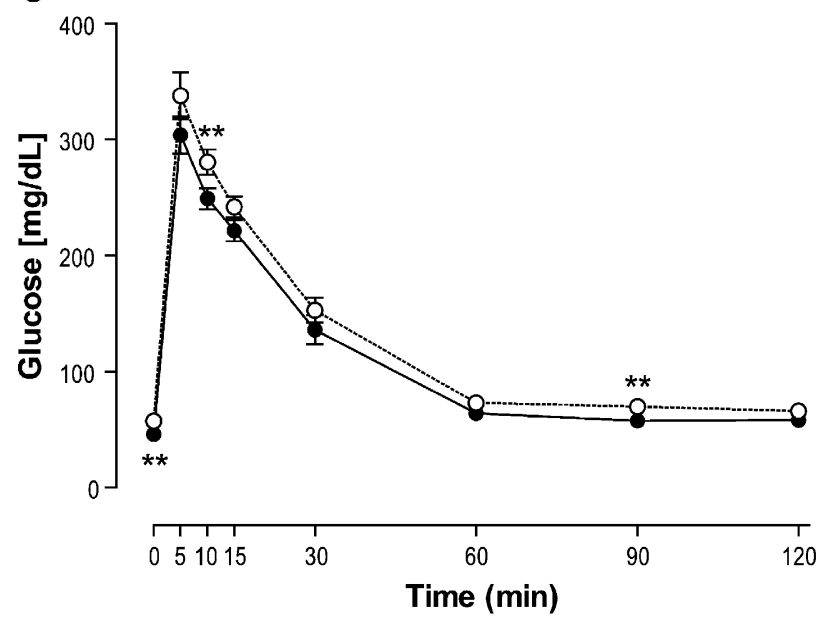

D

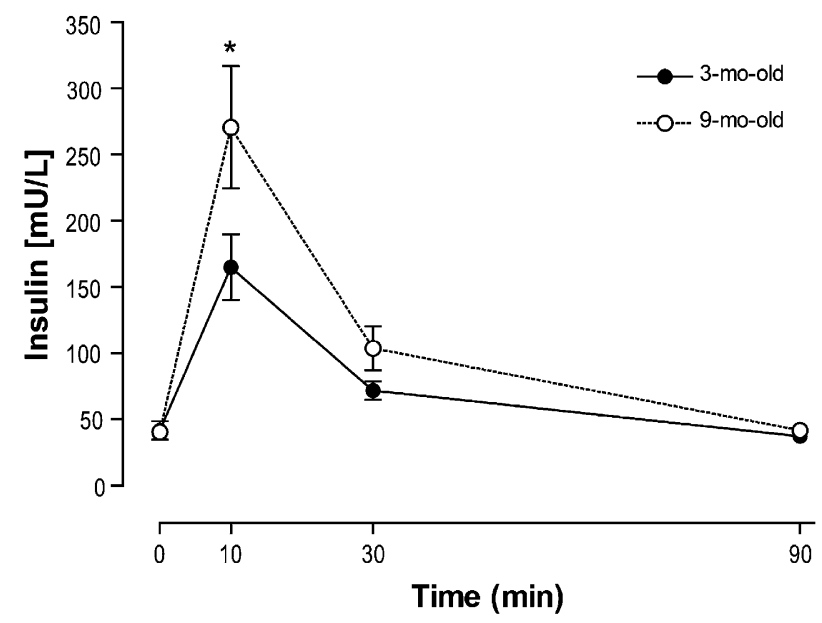

Fig. 1. Blood glucose (A, C) and plasma insulin concentrations (B, D) during IVGTT in nonpregnant (A, B) and pregnant (C, D) rats on day 20 of pregnancy in 9-month-old (dotted line) and 3-month-old rats. Values are means \pm SEM. ${ }^{*} P>.05$ and $P<.1 ; * * P<.05 ; * * * P<.01$ (unpaired $t$ test). Two-factor ANOVA: AUC $_{\text {glucose }}$ is significantly affected by age $(P<.05)$ but not pregnancy, whereas $\operatorname{AUC}_{\text {insulin }}$ is affected by both age $(P<.05)$ and pregnancy $(P<.05$, no interaction between both). 
Table 2

Body composition of pregnant (day 22) and nonpregnant 3- and 9-month-old rats

\begin{tabular}{|c|c|c|c|c|c|c|c|}
\hline & \multicolumn{2}{|c|}{3 mo old } & \multicolumn{2}{|c|}{9 mo old } & \multicolumn{3}{|c|}{ Two-factor ANOVA $(P)$} \\
\hline & $\operatorname{NPr}(\mathrm{n}=8)$ & $\operatorname{Pr}(\mathrm{n}=7)$ & $\mathrm{NPr}(\mathrm{n}=9)$ & $\operatorname{Pr}(\mathrm{n}=9)$ & Age & Pregnancy & Interaction \\
\hline Adipose tissue (g) & $29.4 \pm 2$ & $27.6 \pm 3$ & $42.8 \pm 5$ & $50.9 \pm 4$ & $<.0001$ & NS & NS \\
\hline Adipose tissue $(\%)$ & $11.3 \pm 0.7$ & $9.7 \pm 0.9$ & $14.1 \pm 1.8$ & $14.1 \pm 0.9$ & $<.01$ & NS & NS \\
\hline Lean tissue $(\mathrm{g})$ & $225 \pm 6^{\mathrm{a}}$ & $247 \pm 5^{b}$ & $252 \pm 6.5^{\mathrm{b}}$ & $295 \pm 5^{c}$ & $<.0001$ & $<.0001$ & $<.08$ \\
\hline Lean tissue $(\%)$ & $85.5 \pm 0.8$ & $86.9 \pm 0.9$ & $82.5 \pm 1.5$ & $82.7 \pm 0.9$ & $<.005$ & NS & NS \\
\hline
\end{tabular}

Number of animals is indicated in parentheses. All values are means \pm SEM. For lean tissue, intergroup differences are also denoted with different superscripts according to Bonferroni post hoc test.

The longitudinal data during pregnancy (days 6,13 , and 20) showed that body weight of both 3- and 9-month-old rats increased gradually (ANOVA, both $P<.0001$ ) and that weight gain was comparable in both groups (data not shown). Nonfasting glucose levels dropped in both 3-monthold (ANOVA, $P<.001$ ) and 9-month-old rats (ANOVA, $P<.0001)$ during pregnancy. No significant changes were documented for insulin or leptin concentrations during pregnancy in either 3- or 9-month-old rats, but 9-month-old rats have higher leptin concentrations than 3-month-old rats throughout pregnancy. Analysis of variance indicated small effects of late pregnancy (day 22) on cholesterol (decrease) and triglyceride (increase) concentrations (Table 1). No significant interactions between age and late pregnancy were found, except for nonfasting glucose and insulin, but Bonferroni post hoc test revealed no meaningful differences between late-pregnant animals.

At 10, 15, 30, and 60 minutes during the IVGTT, glucose levels of nonpregnant 9-month-old rats were elevated compared with that of 3-month-old rats (Fig. 1A); the $\mathrm{AUC}_{\text {glucose }}$ in 9-month-old rats was higher than in 3-month-old rats (7539 \pm 433 vs $5731 \pm 537 ; t$ test, $P<.05)$. No differences were detected between the insulin concentrations during the IVGTT of 3- and 9-month-old rats before pregnancy (Fig. 1B). On day 20 of pregnancy, fasting glucose levels were higher in 9-month-old rats than in 3-month-old rats (Fig. 1C); in addition, glucose was elevated at the 10- and 90-minute time points. However, there was no difference in $\mathrm{AUC}_{\text {glucose }}$, whereas the $\mathrm{AUC}_{\text {insulin }}$ showed a tendency toward significance ( $t$ test, $P=.07)$. Two-factor ANOVA indicated that the $\mathrm{AUC}_{\text {glucose }}$ was affected by age $(P<.05)$, but not pregnancy, whereas the $\mathrm{AUC}_{\text {insulin }}$ was influenced by both age $(P<.05)$ and pregnancy $(P<.05)$; no interaction between age and pregnancy was found for either $\mathrm{AUC}_{\text {glucose }}$ or $\mathrm{AUC}_{\text {insulin }}$.

Nine-month-old rats had a higher lean and adipose tissue mass compared with 3-month-old rats (Table 2). A significant correlation was found between body weight and leptin levels in 9-month-old rats (Spearman correlation, $P<.0001 ; r=0.71$ ), but not in 3-month-old rats. On day 22 of pregnancy, lean tissue mass, but not adipose tissue mass, was higher in 9-month-old rats than in 3-month-old rats. No significant interactions were detected.

No differences were detected in litter size or total live litter weight $(50.7 \pm 3.8 \mathrm{~g}[\mathrm{n}=8]$ and $42.2 \pm 4.8 \mathrm{~g}[\mathrm{n}=9]$, respectively) on day 22 of pregnancy between 3- and 9-month-old rats. However, individual fetal weight was reduced by $5 \%$ in 9-month-old rats compared with 3-monthold controls $(4.63 \pm 0.06 \mathrm{~g}[\mathrm{n}=82]$ vs $4.88 \pm 0.04 \mathrm{~g}$ $[\mathrm{n}=80] ; t$ test, $P<.001)$. Placental weight was also decreased in 9-month-old rats $(0.56 \pm 0.01$ vs $0.63 \pm 0.01 \mathrm{~g}$ in 3-month-old rats; $t$ test, $P<.0005)$. In addition, fetal glucose levels were reduced in 9-month-old rats $(83 \pm$ $5.9 \mathrm{mg} / \mathrm{dL}[\mathrm{n}=18]$ vs $111 \pm 5.5 \mathrm{mg} / \mathrm{dL}$ in 3-month-old rats $[\mathrm{n}=14] ; t$ test, $P<.005)$. However, no differences were measured in fetal insulin, cholesterol, triglyceride, and leptin concentrations between the 2 groups. In 9-month-old rats, but not in 3-month-old rats, a significant correlation was detected between maternal and fetal leptin levels (Spearman correlation, $P<.05 ; r=0.88$ ).

\section{Discussion}

This study did not confirm our hypothesis that glucose tolerance would be decreased in 9-month-old pregnant rats. We hypothesized that glucose tolerance during pregnancy is maintained in young rats in the presence of hepatic and peripheral insulin resistance because of adequate beta-cell compensation; we suspected that failing beta-cell function in 9-month-old rats would compromise this compensation. Pregnancy-induced insulin resistance is compensated by increasing basal insulin concentrations in 3-month-old rats during pregnancy, whereas in 9-month-old rats, basal insulin levels decrease during pregnancy. These data, suggesting failing beta-cell function in aging pregnant rats, could not be confirmed during IVGTT; our results showed that the $\mathrm{AUC}_{\text {glucose }}$ during an IVGTT increased with age as expected, but for $\mathrm{AUC}_{\text {insulin }}$ and $\mathrm{AUC}_{\text {glucose }}$ there was no interaction between age and pregnancy. Hence, 9-month-old rats appear to be "coping" in terms of glucose tolerance when insulin resistance is temporarily increased by pregnancy. Although extrapolating these rat data to the human situation is precluded by important species differences, these experiments could suggest that the finding of increased GDM prevalence in women during the late reproductive period (or postmenopause) is largely an age phenomenon per se.

There is good evidence in humans that the insulin resistance of aging is related to increased fat mass $[5,18]$. In line with these data, the removal of visceral fat in 20-month- 
old male rats prevented the insulin resistance and glucose intolerance associated with aging [19]. In this study, we found that 9-month-old rats had increased fat mass on DXA analysis and also increased plasma leptin, cholesterol, and triglyceride concentrations. Previous studies have shown that increased leptin gene expression with age is the result of both higher adiposity and leptin gene expression per unit of adipose tissue [20]. In addition, the decline in hypothalamic leptin sensitivity with aging may be partly independent of fat mass because it was also observed in energy-restricted rats [21]. Insulin stimulates leptin gene expression and secretion in vivo and in vitro, through its trophic effect on adipocytes [22]. In turn, leptin affects insulin secretion through functional receptors on pancreatic beta cells [23], thereby suppressing insulin gene expression and secretion [24,25]. This feedback loop between the adipose tissue and the endocrine pancreas is often referred to as the adipoinsular axis [26]. A dysregulation of the adipoinsular axis was suggested in rats with obesity-induced diabetes [27]; further studies are needed on adipoinsular crosstalk in aging rats.

The age-induced obesity in 9-month-old rats is reflected in typical adiposity-related metabolic parameters, but these parameters do not change dramatically when aging animals become pregnant. During pregnancy, leptin levels in both 3- and 9-month-old rats remained constant up to day 20 , although they were somewhat lower on day 22. A drop in leptin concentrations before parturition was previously documented in rats [28] and in humans [29], whereas the drop in glucose levels at the end of rat pregnancy seems to be a characteristic typical for this species. Interestingly, we found a correlation between maternal and fetal leptin levels on day 22 of pregnancy in 9-month-old rats, suggesting transfer of maternal leptin through the placenta and secretion into the fetal circulation. On immunohistochemistry of rat placentas, leptin is present in the endocrine trophospongium area, and the leptin receptor is present in the labyrinthine region at the lining of the maternal blood vessels, where exchange between maternal and fetal blood occurs (Caluwaerts et al, unpublished data). However, other studies in pregnant rats demonstrated a parallel increase in plasma leptin levels with adipose tissue [30], suggesting that placental leptin contributes little to circulating leptin $[31,32]$.

In 9-month-old rats, reproductive parameters were less optimal. Although litter size and total litter weight were not significantly altered, there was a $5 \%$ reduction in average individual fetal weight and a $13 \%$ decrease in placental weight. These effects could be the result of impaired uteroplacental blood flow [33]. Aging in rodents probably impairs uteroplacental blood flow; indeed, uterine arteries of 9-month-old rats were shown to have an impaired reactivity [34], and the remodeling of uterine arteries was compromised in 40-week-old pregnant mice compared with 12-week-old mice [35]. In the experiments as we described them, uteroplacental blood flow was not examined; thus, the relevance of this $5 \%$ decrease in fetal weight needs further examination.

In conclusion, 9-month-old rats exhibited a deterioration in glucose tolerance, an increase in fat mass, and elevated leptin concentrations. Pregnancy did not aggravate these parameters, indicating that the impaired glucose tolerance observed in 9-month-old pregnant rats is an age-related phenomenon. Our data could suggest that the increased prevalence of glucose intolerance in older women may be explained by age as such. However, important species differences preclude the extrapolation of these rat data to the human situation; further investigation of the effect of age on human pregnancy seems therefore necessary.

\section{Acknowledgment}

S Caluwaerts and this project were supported by a grant from the Katholieke Universiteit Leuven (Verkennende Internationale Samenwerking, ZKA 5850).

\section{References}

[1] Ventura SJ, Hamilton BE, Sutton PD. Revised birth and fertility rates for the United States, 2000 and 2001. Natl Vital Stat Rep 2003;51: [National Center for Health Statistics].

[2] Prysak M, Lorenz RP, Kisly A. Pregnancy outcome in nulliparous women 35 years and older. Obstet Gynecol 1995;85:65-70.

[3] Solomon CG, Willett WC, Carey VJ, et al. A prospective study of pregravid determinants of gestational diabetes mellitus. JAMA 1997;278:1078-83.

[4] Paulson RJ, Boostanfar R, Saadat P, et al. Pregnancy in the sixth decade of life; obstetric outcomes in women of advanced reproductive age. JAMA 2002;288:2320-3.

[5] Basu R, Breda E, Oberg AL, et al. Mechanisms of the age-associated deterioration in glucose tolerance: contribution of alterations in insulin secretion, action, and clearance. Diabetes 2003;52:1738-48.

[6] Iozzo P, Beck-Nielsen H, Laakso M, et al. Independent influence of age on basal insulin secretion in nondiabetic humans. J Clin Endocrinol Metab 1999;84:863-8.

[7] Escriva F, Agote M, Rubio E, et al. In vivo insulin-dependent glucose uptake of specific tissues is decreased during aging of mature Wistar rats. Endocrinology 1997;138:49-54.

[8] Novelli M, De Tata V, Bombara M, et al. Age-dependent reduction in GLUT-2 levels is correlated with the impairment of the insulin secretory response in isolated islets of Sprague-Dawley rats. Exp Gerontol 2000;35:641-51.

[9] Remacle C, De Clercq L, Delaère P, et al. Organ culture of islets of Langerhans from young and senescent rats. Cell Tissue Res 1980;207: 429-48.

[10] Lam NT, Cheung AT, Riedel MJ, et al. Leptin reduces glucose transport and cellular ATP levels in INS-1 beta-cells. J Mol Endocrinol 2004;32:415-24.

[11] Tsiotra PC, Tsigos C, Raptis SA. TNFalpha and leptin inhibit basal and glucose-stimulated insulin secretion and gene transcription in the HIT-T15 pancreatic cells. Int J Obes Relat Metab Disord 2001;25: $1018-26$

[12] Freinkel N. Banting lecture 1980. Of pregnancy and progeny. Diabetes 1980;29:1023-35.

[13] Xiang AH, Peters RK, Trigo E, et al. Multiple metabolic defects during late pregnancy in women at high risk for type 2 diabetes. Diabetes 1999;48:848-54. 
[14] Leturque A, Burnol AF, Ferré P, et al. Pregnancy-induced insulin resistance in the rat: assessment by glucose clamp technique. Am J Physiol 1984;246:E25 -E31.

[15] Bone AJ, Taylor KW. Metabolic adaptations to pregnancy shown by increased biosynthesis of insulin in islets of Langerhans isolated from pregnant rats. Nature 1976;262:501-2.

[16] Kalkhoff RK, Kim HJ. Effects of pregnancy on insulin and glucagon secretion by perifused rat pancreatic islets. Endocrinology 1978;102: $623-31$.

[17] Aerts L, Van Assche FA. Ultrastructural changes of the endocrine pancreas in pregnant rats. Diabetologia 1975;11:285-9.

[18] Kohrt WM, Kirwan JP, Staten MA, et al. Insulin resistance in aging is related to abdominal obesity. Diabetes 1993;42:273-81.

[19] Gabriely I, Ma XH, Yang XM, et al. Removal of visceral fat prevents insulin resistance and glucose tolerance of aging. Diabetes 2002;51: $2951-8$.

[20] Li H, Matheny M, Nicolson M, et al. Leptin gene expression increases with age independent of increasing adiposity in rats. Diabetes 1997;46:2035-9.

[21] Gabriely I, Ma XH, Yang XM, et al. Leptin resistance during aging is independent of fat mass. Diabetes 2002;51:1016-21.

[22] Kolaczynski JW, Nyce MR, Considine RV, et al. Acute and chronic effects of insulin on leptin production in humans: studies in vivo and in vitro. Diabetes 1996;45:699-701.

[23] Kieffer TJ, Heller RS, Habener JF. Leptin receptors expressed on pancreatic beta-cells. Biochem Biophys Res Commun 1996;224: $522-7$.

[24] Emilsson V, Liu YL, Cawthorne MA, et al. Expression of the functional leptin receptor mRNA in pancreatic islets and direct inhibitory action of leptin on insulin secretion. Diabetes 1997;46:313-6.

[25] Seufert J, Kieffer TJ, Leech CA, et al. Leptin suppression of insulin secretion and gene expression in human pancreatic islets: implications for the development of adipogenic diabetes mellitus. J Clin Endocrinol Metab 1999;84:670-6.
[26] Kieffer TJ, Habener JF. The adipoinsular axis: effects of leptin on pancreatic $\beta$-cells. Am J Physiol Endocrinol Metab 2000;278: E1-E14.

[27] Vickers MH, Reddy S, Ikenasio BA, et al. Dysregulation of the adipoinsular axis - a mechanism for the pathogenesis of hyperleptinemia and adipogenic diabetes induced by fetal programming. J Endocrinol 2001;170:323-32.

[28] Stocker C, O’Dowd J, Morton NM, et al. Modulation of susceptibility to weight gain and insulin resistance in low birthweight rats by treatment of their mothers with leptin during pregnancy and lactation. Int J Obes 2004;28:129-36.

[29] Masuzaki H, Ogawa Y, Sagawa N, et al. Nonadipose tissue production of leptin: leptin as a novel placenta-derived hormone in humans. Nat Med 1997;3:1029-33.

[30] Herrera E, Lasuncion MA, Huerta L, et al. Plasma leptin levels in rat mother and offspring during pregnancy and lactation. Biol Neonate 2000;78:315-20.

[31] Tereda Y, Yamakawa K, Sugaya A, et al. Serum leptin levels do not rise during pregnancy in age-matched rats. Biochem Biophys Res Commun 1998;253:841-4

[32] Kawai M, Yamaguchi M, Murakami T, et al. The placenta is not the main source of leptin production in pregnant rat: gestational profile of leptin in plasma and adipose tissues. Biochem Biophys Res Commun 1997;240:798-802.

[33] Wentzel P, Jansson L, Eriksson UJ. Diabetes in pregnancy: uterine blood flow and embryonic development in the rat. Pediatr Res 1995;38:598-606.

[34] Wight E, Küng CF, Moreau P, et al. Aging, serum estradiol levels, and pregnancy differentially affect vascular reactivity of the rat uterine artery. J Soc Gynecol Investig 2000;7:106-13.

[35] van der Heijden OW, Essers YP, Simkens LH, et al. Aging blunts remodeling of the uterine artery during murine pregnancy. J Soc Gynecol Investig 2004;11:304-10. 